\title{
Unravelling the 'Uteropolitic': Problematizing the Global Family Planning Apparatus through a Post-Colonial Feminist Lens from Pakistan
}

\author{
Muhammad Moiz ${ }^{1}$, Maryam Khan ${ }^{2}$, and Muneeb Ahmad ${ }^{3}$ \\ ${ }^{1}$ Program Manager, Global Health Directorate, Indus Health Network, Karachi, Pakistan \\ ${ }^{2}$ Community Engagement Centre, Joint Venture of Global Health Directorate, Indus Health \\ Network (IHN - GHD), Karachi, Pakistan \\ ${ }^{3}$ Interactive Research and Development (IRD), Karachi, Pakistan
}

\begin{abstract}
Using Pakistan as a case study, in this inductive essay we theorize that family planning (FP) in the "developing" world emerges as a complex bio-political system of patriarchal reproductive governance. Our discourse starts with one problematic observation: "family planning", despite its holistic conceptualisation, has become synonymous with "contraception". Reproductive governance in post-colonial states has long deployed a simultaneously moralistic and utilitarian "population control" narrative. Consequently, public health programming for family planning adapted population-level epidemiological constructs such as Contraceptive Prevalence Rate to measure "success"; turning contraceptive use into use into a "desirable" numeric goal. Simultaneously, the global governance and financing of FP, following a neo-liberal market paradigm, has turned reproductive health into a tradeable commodity (contraceptives) between buyers (women) and suppliers (industries and governments) and only available through a market exchange (healthcare services). But because reproduction in patriarchies is a woman's socio-cultural "responsibility", this biopolitical push is enacted on the bodies of women disproportionately, hence perpetuating the patriarchy. Given the obvious complexity of this problem environment, we argue against the default centrality of epidemiology in global reproductive health and call for a radical new epistemology of family planning. We attempt to explain this complexity through general systems theory anchoring ourselves to feminist exegesis. We utilize the concepts of social free energy, social entropy, and social temperature as they apply to complex social systems to re-examine this perpetual biopolitics of patriarchy. We hypothesize that the family, healthcare, markets, and governments collectivize as social institutions to create a complex system of reproductive governance that expends women's agency and well-being as resources to preserve its entropic state(s) of patriarchal hegemony. We conclude with various feminist ruminations on what our model represents, and speculate what the model can look like in a reproductive utopia.
\end{abstract}

Keywords: Biopolitics; family planning; contraceptives; population control; complexity; patriarchy; reproductive justice; reproductive governance 